\title{
The value of plant extracts with antioxidant activity in attenuating coccidiosis in broiler chickens
}

\author{
V. Naidoo ${ }^{a}$, L.J. McGaw ${ }^{a}$, S.P.R. Bisschop ${ }^{b}$, N. Duncan ${ }^{a}$ and J.N. Eloff ${ }^{a}$ \\ aDepartment of Paraclinical Sciences, Faculty of Veterinary Science, University of Pretoria, \\ South Africa
}

${ }^{b}$ Department of Production Animal Studies, Faculty of Veterinary Science, University of Pretoria, South Africa

\begin{abstract}
Coccidiosis remains one of the most important diseases in the poultry industry and results in the annual loss of millions of US dollars by the poultry industry. In South Africa and other developing countries where a large percentage of the population is unemployed, cheap food production is necessary. If the control of the coccidian parasite could be made more economical, these savings could be passed on to the consumer. In Europe, where the economics are different, people are becoming more aware of the potential dangers of using antimicrobials in producing animal protein. A solution to both these problems could be the use of plant products that function by mechanisms other than those of chemotherapeutics, with the additional advantage of a natural origin. Antioxidant compounds could hold promise for the control of Eimeria infections due to the association of coccidial infection with lipid peroxidation of the intestinal mucosa. Four plant extracts with antioxidant activity were screened for their anticoccidial activity in vivo with toltrazuril as the positive control. Combretum woodii $(160 \mathrm{mg} / \mathrm{kg}$ ) proved to be extremely toxic to the birds, while treatment with Tulbaghia violacea $(35 \mathrm{mg} / \mathrm{kg})$, Vitis vinifera $(75 \mathrm{mg} / \mathrm{kg})$ and Artemisia afra $(150 \mathrm{mg} / \mathrm{kg})$ resulted in feed conversion ratios similar to toltrazuril, and higher than the untreated control. $T$. violacea also significantly decreased the oocyst production in the birds. From this study we conclude that antioxidant-rich plant extracts have potential benefits in treating coccidial infections. The promising results obtained with $T$. violacea justify further studies on the potential value of the plant as a therapeutic or prophylactic anticoccidial agent.
\end{abstract}

Keywords: Anticoccidial; Poultry; Herbal remedies; Ethnoveterinary medicines; Artemisia afra; Grape seed extract; Tulbaghia violacea; Combretum woodii

\section{Introduction}

The South African poultry industry is the largest contributor to the agricultural economy with approximately 12 million broiler chickens being slaughtered per week (Germishuis, 2006). In a country with high unemployment rates, this industry is a very important supplier of relatively low cost protein to all sectors of the South African economy (Statistics SA, 2006). One of the major problems facing the commercial poultry industry is coccidiosis (unpublished data obtained from the South African Poultry Industry).

Although the actual losses incurred in South Africa remain unknown, they are believed to run into millions of dollars due to resultant production losses. In the United Kingdom, to which South Africa compares favourably in terms of production capacity and systems, the total cost 
of coccidiosis in chickens was estimated at US $\$ 77$ million in 1995 and resulted from a combination of in-food medication costs, veterinary costs and production losses (Williams, 1999). At present South African commercial poultry farmers spend approximately US $\$ 0.02$ per bird (US $\$ 12.5$ million annually) on the use of in-feed prophylactic anticoccidials in order to limit mortalities and enhance broiler growth and production. The anticoccidials in use include the polyether (ionophor) group of chemotherapeutics, sulphonamides, pyrimidine derivatives, triazinetriones and the benzenacetonitriles (Carrington et al., 2007).

Unfortunately, with the widespread use of anticoccidial drugs, resistance has developed to all the drugs introduced thus far ([Chapman, 1997] and [Chapman, 1998]). An additional constraint in their use comes from the consumer and the ever-increasing need for the drugfree production of foods (Harper and Makatouni, 2002). The use of plant extracts as medicants may alleviate these difficulties, as they are not only natural products but may comprise new therapeutic molecules to which resistance has not yet developed. The use of herbal remedies in the management of coccidiosis is not a new concept. For example, halofuginone, a quinazolinone alkaloid derived from Dichroa febrifuga, has been used as a coccidiostat, and the original extract from $D$. febrifuga, known as febrifugine, possesses antimalarial and anticoccidial activity (Youn and Noh, 2001). The investigation of herbal materials as anticoccidial remedies therefore holds promise as an alternate in the control of coccidiosis. In addition to possibly lowering the cost of food production in South Africa, it could create a herbal remedy export market and thereby create more jobs in the country. The use of herbal extracts may also satisfy the increasing concerns of consumers, on condition that they prove to be both safe and effective. South Africa possesses an enormous diversity of plant resources, which has yielded many examples of useful bioactive compounds ([Van Wyk, 2002] and [Fennell et al., 2004]). We discuss the in vivo effect of four herbal extracts prepared from South African plants on artificially induced coccidial infection in 2-week-old broiler chickens.

For this study Combretum woodii Dummer (Combretaceae), Vitis vinifera L. (Ampelidaceae), Artemisia afra Jacq. ex Willd. (Asteraceae) and Tulbaghia violacea Harv. (Liliaceae) were selected on the basis of previously reported antioxidant activity ([Allen et al., 1998], [Burits et al., 2001], [Kubec et al., 2002], [Zishiri, 2004], [Chikoto and Eloff, 2005], [Zheng and Wang, 2001] and [Velíšek et al., 2006]). According to Allen et al. (1998) antioxidant compounds are known to reduce the severity of Eimeria tenella infections by ameliorating the degree of intestinal lipid peroxidation. One of the most potent veterinary anticoccidials, toltrazuril, is believed to achieve some, if not all, of its beneficial effect by limiting the degree of lipid peroxidation (Eraslan et al., 2004).

\section{Materials and methods}

\subsection{Plant collection and preparation}

A. afra, C. woodii and T. violacea were collected in Pretoria, South Africa, in September 2005. Voucher specimens were deposited in the Herbarium of the Department of Paraclinical Sciences (Medicinal Plant Collection) for A. afra (voucher number McGaw200502), C. woodii (voucher number McGaw200503) and T. violacea (voucher number McGaw200504), respectively. The leaves and twigs of $A$. afra, leaves of $C$. woodii, and roughly chopped whole plants of $T$. violacea were dried at $40{ }^{\circ} \mathrm{C}$ in a forced stream of air using a custom-built plant 
drying machine. The plant material was ground to a powder using a Macsalab Model $200 \mathrm{LAB}$ grinder. Extracts were prepared by maceration with shaking (Labotec Model 20.2 shaker) for $24 \mathrm{~h}$ in $70 \%$ acetone with a 10:1 solvent to dry weight ratio (Eloff, 1998). The extracts were filtered through Whatman No 1 filter paper using a Büchner funnel, and the acetone was removed using a rotary evaporator (Büchi Rotavapor R-200). An aliquot of the remaining aqueous plant extract for each species was freeze-dried (Specht Scientific freeze-drier) to determine the concentration of the plant extracts prior to use in the anticoccidial assay.

$V$. vinifera (GS) extract was prepared from seeds according to a patented method developed to maximise antioxidant capacity (Chikoto and Eloff, 2005). Briefly, oil-expressed grape seed (a waste product from grape seed oil production) was washed with water and the marc extracted with $80 \%$ ethanol. The extract was filtered and passed through Diaion HP20 and dried to obtain an antioxidant-rich extract. In preparation for the anticoccidial assay, the grape seed extract powder was dissolved in a small volume of $60 \%$ ethanol and the volume subsequently increased with distilled water so that the final concentration of ethanol was $12 \%$. The concentration represented the point of maximum solubility of the extract in water.

To assess the stability of the extracts, aliquots $(100 \mu \mathrm{g})$ of the extracts were separated using thin layer chromatography (TLC, Merck aluminium-backed plates, silica gel $60 \mathrm{~F}_{254}$ ). Three solvent systems of differing polarities were used (Kotzé and Eloff, 2002), namely ethyl acetate:methanol:water (40:5.4:5, EMW, polar, neutral), chloroform:ethyl acetate:formic acid (5:4:1, CEF, intermediate polarity, acidic) and benzene:ethanol:ammonium hydroxide (90:10:1, BEA, non-polar, basic). The extracts were analysed using TLC immediately after preparation, and again before use in the anticoccidial screening. In the interim, the extracts were stored in the dark in a cold room at $4{ }^{\circ} \mathrm{C}$. The TLC fingerprints before and after storage were compared to assess potential degradation or other changes in chemical composition of the extracts.

To confirm the in vitro antioxidant scavenging capacity of the selected plants in comparison to toltrazuril, the trolox equivalent antioxidant assay was utilised. For this assay the method of Re et al. (1999) which makes use of a pre-generated radical monocation, 2,2'-azinobis-(3ethylbenzothiazoline-6-sulphonic acid) $\left(\mathrm{ABTS}^{+}\right)$(Sigma-Aldrich, SA) and trolox (water-soluble vitamin $E$ analogue) (Sigma-Aldrich, SA) was adapted (Naidoo et al., 2006). The blue/green chromophore $\mathrm{ABTS}^{+}$was produced through the reaction between $\mathrm{ABTS}$ and potassium sulphate (Sigma-Aldrich, SA). In this assay the antioxidant activity of a molecule/extract is related to that of trolox, by means of trolox equivalent antioxidant capacity (TEAC) values.

\subsection{Animals and management}

Broiler chickens were reared as a single group from day old to 2 weeks on wire mesh. The animals (492 $\pm 20 \mathrm{~g}$ ) were subsequently randomly divided by tag-number into treatment groups, viz. herbal extracts $(n=15)$ per group, toltrazuril (Rx, $n=15)$, a non-treated infected $(\mathrm{I}, n=20)$ and non-treated non-infected group $(\mathrm{C}, n=20)$ with each group being allocated to two large cages with a single tray per group to catch faecal material. The birds had free access to water and a commercial broiler feed formulated without any anticoccidial medication ([Youn and Noh, 2001], [Christaki et al., 2004] and [Du and Hu, 2004]). The birds were housed in a closed house under negative pressure ventilation with an artificial light source. Feed intake and weight gain for each group was determined weekly from 14 days until the day of infection and subsequently every alternate day as far as practical. 
The birds were infected with $0.2 \mathrm{ml}$ of a mixed suspension of Eimeria tenella $(80 \%), E$. maxima $(9 \%)$ and $E$. avervulina (11\%). containing 500000 viable oocysts per milliliter. The chickens were monitored daily for signs of infection, which was defined as the presence of oocysts in the faeces with concurrent bloody diarrhoea (Holdsworth et al., 2004). At the first signs of infection, chickens were treated with one of the herbal extracts, once a day for 5 days at $1 \mathrm{ml} / \mathrm{bird}$ by gavage. A treatment period of 5 days was selected as this was the estimated period of oxidant insult induced by the coccidian parasite (Koinarski et al., 2005) The negative control birds received no treatment while the positive control birds were treated with toltrazuril at the recommended dose of $20 \mathrm{mg} / \mathrm{kg}$ per os for 2 days.

\subsection{Observations and analytical procedures}

All statistical analysis was undertaken using the programme SPSS 13 (SPSS Inc.). Pooled oocyst counts, per group, were undertaken as previously described by Holdsworth et al. (2004). Individual oocyst counts were not feasible due to the inability to house birds individually. In total $10 \mathrm{~g}$ of faeces was collected and processed. The changes in oocysts per gram (OPG) over time were evaluated using the paired sample $t$-test.

The mean weight gain (MWG) was calculated using the formula: $M W G=$ (mean final weight of live birds in a cage or pen) - ((mean initial weight of all birds in that cage or pen) + (weight of dead birds)). Differences in the average weights per group for each time point were compared using ANOVA to determine if a significant difference was present between the treatment groups. Weight gains for each group for the different time points were compared using a paired sample $t$-test to ascertain if weights differed significantly over the growth period.

The group feed conversion ratio (FCR) for the study period was calculated using the formula: $\mathrm{FCR}=$ feed consumed per group $(\mathrm{g}) /$ (weight gain of surviving birds + weight gain of dead birds). Individual FCR were not calculated as animals were fed as a group. Changes in FCR over time were evaluated using the paired $t$-test.

The mortality rate was determined using the formula: percent mortality $=$ (total number of dead chicks in the cage/initial number of birds in cage) $\times 100$ and evaluated for death rate and hazard analysis after being exposed to the infectious agent in the absence and presence of the herbal extract. Necropsies were performed on all mortalities. Five birds from the control group were euthanised at the first signs of infection by carbon dioxide inhalation. Full postmortem examinations and histopathological scoring of lesions was done. Five birds were subsequently euthanased on day 32 and the remaining birds at 39 days of age. After slaughter the intestinal tract was examined and scored using a score of 0-4 (Johnson and Reid, 1970).

\section{Results}

All the plant extracts showed adequate antioxidant activity, with toltrazuril being more effective than the plant extracts (TEAC $=1.6$ ). TEAC values in decreasing order were 1.2, $0.75,0.5$ and 0.2 for Grape seed extract, $A$. afra, C. woodii and $T$. violacea, respectively. 
The final concentrations of plant extract administered to the chickens were $210 \mathrm{mg} / \mathrm{ml}$ (160 mg/kg) (C. woodii), $200 \mathrm{mg} / \mathrm{ml}(75 \mathrm{mg} / \mathrm{kg})(\mathrm{GS}), 100 \mathrm{mg} / \mathrm{ml}(150 \mathrm{mg} / \mathrm{kg})($. afra) and $51 \mathrm{mg} / \mathrm{ml}(35 \mathrm{mg} / \mathrm{kg})(T$. violacea). These were designated as the maximum concentration of prepared extract soluble in water. TLC analysis revealed no noteworthy changes in the chemical composition of the extracts during storage at $4{ }^{\circ} \mathrm{C}$ until administration to the chickens.

Once infected it took approximately 5 days for the animals to demonstrate clinical signs of infection, which was mild. The necropsy confirmed the low virulence of infectivity with only a few of the infected animals displaying macroscopically visible lesions. Microscopically the lesions varied in their severity with no consistency between any of the treated animals.

When comparing the weight gains between the various times, the animals treated with $C$. woodii were the most severely affected, with the MWG (Fig. 1) differing significantly to all the other groups from the start of clinical signs of infection to euthanasia. No differences were present among $A$. afra, GS, T. violacea and Rx from the time of clinical signs to euthanasia even though they all differed significantly from $\mathrm{C}$ and $\mathrm{I}$. At the termination of the study (39 days) the average weight of a chicken per group was 1.8, 2.1, 2.0, 2.0, 2.0, 2.1 and 2.7 for $C$. woodii, A. afra, GS, T. violacea, Rx, I and C groups, respectively.

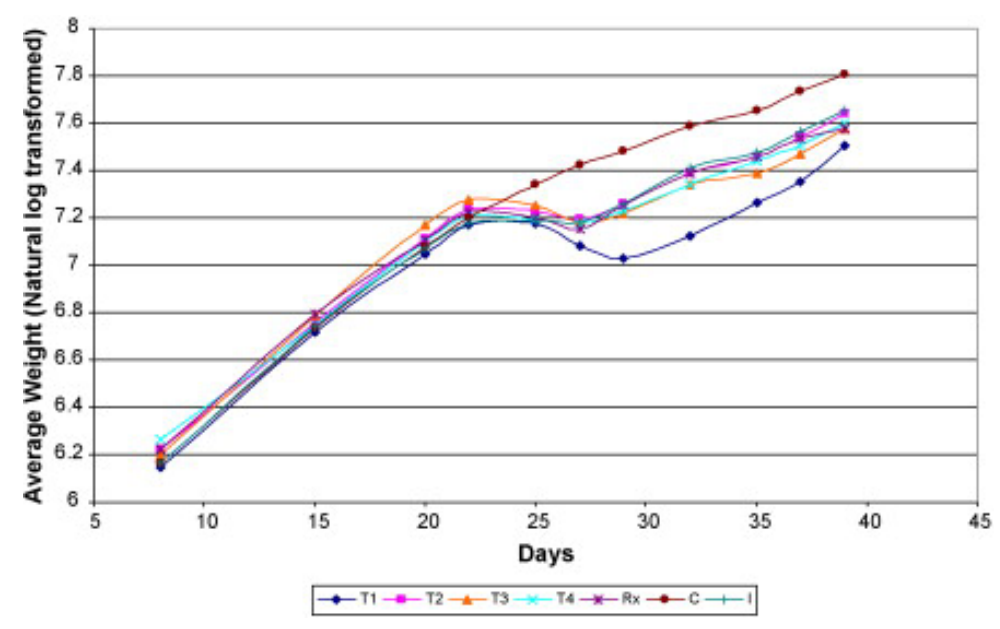

Fig. 1. Average weight gains per treatment group over time. T1: C. woodii; T2: A. afra; T3: Grape seed; T4: $T$. violacea; I: infected non-treated; Rx: toltrazuril, C: non-treated noninfected. Birds were infected on day 21, treated on day 27-31.

When comparing the FCR between the groups the animals in the control group had a ratio of 1.94 for the study. The FCR was poorer for all infected animals from the time of infection to recovery, i.e. all birds lost weight while infected. The $C$. woodii group had the poorest FCR of 6.6 which remained poor until euthanasia. When comparing the other treatment groups to the FCR of 2.9 for I, GS and Rx had a similar FCR of 2.46, which was marginally poorer than 2.36 
for $A$. afra and 2.43 for $T$. violacea. Although the FCRs in general appear poor, this was influenced by the greater wasting by the birds being in cages and not on the floor.

From the OPG of faeces (Fig. 2), Rx decreased the OPG after the recommended duration of treatment and was superior to the effects of the plant extracts. Once treatment was stopped, the OPG increased in the treatment group transiently before decreasing at the same rate as the control. For the plant extracts, only $T$. violacea differed significantly from I. In addition to decreasing the OPG moderately, the lower levels were maintained for the duration of treatment, and increased on cessation of therapy.

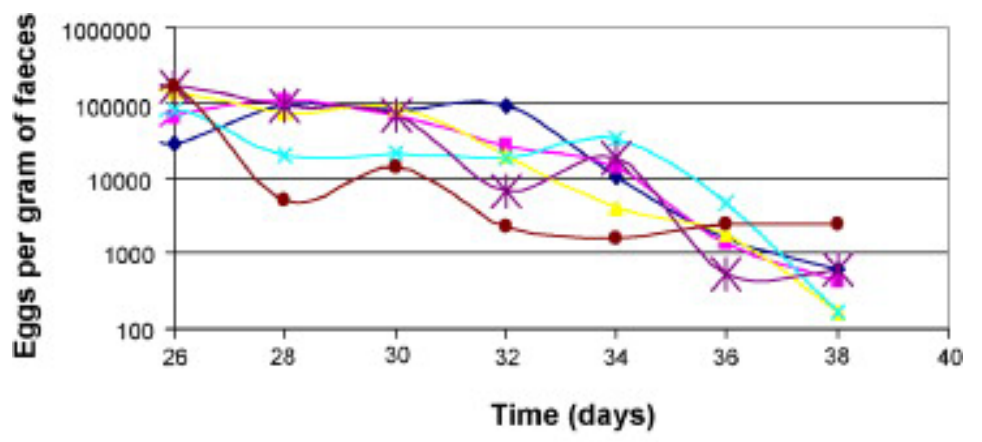

$\rightarrow-\mathrm{T} 1 \rightarrow-\mathrm{T} 2 \rightarrow \mathrm{T} 3 \rightarrow-\mathrm{T} 4 \rightarrow-\mathrm{I} \rightarrow-\mathrm{T} 5$

Fig. 2. Oocyst production over time for each of the treatment groups (natural logarithm transformed). T1: C. woodii; T2: A. afra; T3: grape seed; T4: T. violacea; I: infected nontreated; Rx: toltrazuril. Birds were infected on day 21, treated on day 27-31.

Mortalities were 6 and 1 for $C$. woodii and I, respectively with the mean survival time being 2 days shorter for animals receiving $C$. woodii compared to the other groups. $C$. woodii-treated birds also had the greatest hazard of dying. No mortalities were seen in the other groups.

\section{Discussion}

In all cases, the herbal extracts produced an improved FCR over the control in a similar manner to that for toltrazuril. In addition, $T$. violacea also marginally reduced oocyst shedding in the infected birds. This therefore supports previous findings on the importance of antioxidant compounds in the management of coccidiosis (Allen et al., 1998). With the coccidian parasite-induced host cell destruction being associated with oxidative stress and lipid peroxidation, the antioxidant which have the ability to neutralise reactive oxygen species, are protective due to their ROS scavenging abilty. In the case of $T$. violacea, the antioxidant compounds have been identified as $S$-(methylthiomethyl)cysteine sulfoxide (marasmine), bis[(methylthio)methyl] disulfide and various derivatives ([Zheng and Wang, 2001], [Dictionary of Natural Products, 2006] and [Velíšek et al., 2006]). Grape seeds contain many compounds with antioxidant activity, including monomeric flavanols (catechin and epicatechin), dimeric, 
trimeric and polymeric procyanidins, and phenolic acids (gallic acid and ellagic acid) ([Yilmaz and Toledo, 2004] and [Naidoo et al., 2006]).

The inability of the other plant extracts to reduce the OPG as described for toltrazuril is not known at this stage. Biophasic availability may be the problem as the extracts were administered in water firstly as it is a non-toxic solvent, and secondly to minimise systemic exposure, i.e. lower the bioavailability. With the coccidial parasite being intracellular, a degree of lipid solubility would appear to be important. Toltrazuril is very lipid soluble and is likely to achieve a higher intracellular concentration than water-soluble compounds such as catechin in grape seed. It should also be noted that marasmine, the main antioxidant in $T$. violacea, is also lipid soluble.

Irrespective of the treatments, with the exception of $C$. woodii, the birds recovered at the same rate. The natural recovery of the birds was expected as no re-infection could occur with birds not being in direct contact with their faeces. It was interesting to note that irrespective of treatment, all birds stopped shedding oocysts at the same point, suggesting that this was related more to the life cycle of the parasite rather than to the treatment.

With the poor growth and increased mortality caused by C. woodii we suspect plant toxicity. At present no specified toxicant has been reported to occur in the plant. This therefore appears to be the first case of Combretum toxicity that has been reported in birds.

\section{Conclusions}

With the three plant extracts promoting a similar FCR to toltrazuril, the use of antioxidantcontaining plants may be of benefit in the management of coccidiosis. It is important to note that the active plant constituents should be sufficiently lipid soluble to penetrate intracellularly to have an effect on the coccidian parasite. $T$. violacea displayed a beneficial effect on the rate of oocyst shedding, showing the most promise of all the plant extracts tested in this study. This provides a strong rationale for further evaluation of the anticoccidial efficacy of Tulbaghia violacea plant extracts in a larger study. This plant may also have merit in the prophylactic management of coccidiosis.

\section{Acknowledgements}

The Claude Leon Foundation and the University of Pretoria are thanked for financial support (LJM). The project was funded by the National Research Foundation of South Africa (NRF).

\section{References}

Allen et al., 1998 P.C. Allen, H.D. Danforth and P.C. Augustine, Dietary modulation of avian coccidiosis, Int. J. Parasitol. 28 (1998), pp. 1131-1140.

Burits et al., 2001 M. Burits, K. Asres and F. Bucar, The antioxidant activity of the essential oils of Artemisia afra, Artemisia abyssinica and Juniperus procera, Phytother. Res. 15 (2001), pp. 103-108. 
Carrington et al., 2007 C. Carrington, A. du Plessis and V. Naidoo, Index of Veterinary Specialities, Johncom publishers, Johannesburg (2007) pp. 38-40.

Chapman, 1997 H.D. Chapman, Biochemical, genetic and applied aspects of drug resistance in Eimeria parasites of the fowl, Avian Pathol. 26 (1997), pp. 221-244.

Chapman, 1998 H.D. Chapman, Evaluation of the efficacy of anticoccidial drugs against Eimeria species in the fowl, Int. J. Parasitol. 28 (1998), pp. 1141-1144.

Chikoto and Eloff, 2005 Chikoto, H., Eloff, J.N., 2005. Antioxidant. Patent NR 2005/09681. Application date, November 30, 2005.

Christaki et al., 2004 E. Christaki, P. Florou-Paneri, I. Giannenas, M. Papazahariadou, N.A. Botsoglou and A.B. Spais, Effect of a mixture of herbal extracts on broiler chickens infected with Eimeria tenella, Anim. Res. 53 (2004), pp. 137-144.

Dictionary of Natural Products, 2006 Dictionary of Natural Products, CD-ROM Version 15:1, Chapman and Hall, CRC (2006).

Du and Hu, $2004 \mathrm{~A}$. Du and S. Hu, Effects of a herbal complex against Eimeria tenella infections in chickens, J. Vet. Med. B 51 (2004), pp. 194-197.

Eraslan et al., 2004 G. Eraslan, Y. Cam, M. Eren and B.C. Liman, Changes in malondialdehyde level and catalase activity and effect of toltrazuril on these parameters in chicks infected with Eimeria tenella, Bull. Vet. Inst. Pulawy 48 (2004), pp. 251-254.

Fennell et al., 2004 C.W. Fennell, K.L. Lindsey, L.J. McGaw, S.G. Sparg, G.I. Stafford, E.E. Elgorashi, O.M. Grace and J. van Staden, Assessing African medicinal plants for efficacy and safety: pharmacological screening and toxicology, J. Ethnopharmacol. 94 (2004), pp. 205217. Germishuis, 2006 Germishuis, H., 2006. South Africa, Poultry and Products. GAIN Report, SF6033, US Embassy, Pretoria, 11 pp.

Harper and Makatouni, 2002 C.G. Harper and A. Makatouni, Consumer perception of organic food production and farm animal welfare, Br. Food J. 104 (2002), pp. 287-299.

Holdsworth et al., 2004 P.A. Holdsworth, D.P. Conway, M.E. McKenzie, A.D. Dayton, H.D. Chapman, G.F. Mathis, J.T. Skinner, H.C. Mundt and R.B. Williams, World Association for the Advancement of Veterinary Parasitology (WAAVP) guidelines for evaluating the efficacy of anticoccidial drugs in chickens and turkeys, Vet. Parasitol. 121 (2004), pp. 189-212.

Johnson and Reid, 1970 J. Johnson and W.M. Reid, Anticoccidial drugs: lesion scoring techniques in battery and floor-pen experiments with chickens, Exp. Parasitol. 28 (1970), pp. 30-36.

Kotzé and Eloff, 2002 M. Kotzé and J.N. Eloff, Extraction of antibacterial compounds from Combretum microphyllum (Combretaceae), S. Afr. J. Bot. 68 (2002), pp. 62-67. 
Koinarski et al., 2005 V. Koinarski, N. Georgieva, V. Gadjeva and P. Petkov, Antioxidant status of broiler chickens infected with Eimeria acervulina, Rev. Méd. Vét. 156 (10) (2005), pp. 498-502.

Kubec et al., 2002 R. Kubec, J. Velíšek and R.A. Musah, The amino acid precursors and odor formation in society garlic (Tulbaghia violacea Harv.), Phytochemistry 60 (2002), pp. 21-25.

Naidoo et al., 2006 V. Naidoo, H. Chikoto, L.C. Bekker and J.N. Eloff, Antioxidant compounds in Rhoicissus tridentata extracts may explain their antibabesial activity, S. Afr. J. Sci. 102 (2006), pp. 198-201.

Re et al., 1999 R. Re, N. Pellegrini, A. Proteggente, A. Pannala, M. Yang and C. Rice-Evans, Antioxidant activity applying an improved ABTS radical cation decolorization assay, Free Radical Biol. Med. 26 (1999), pp. 1231-1237.

Statistics SA, 2006 Statistics SA, 2006. Labour force survey, Statistics South Africa, Private Bag X44, Pretoria 0001.

Van Wyk, 2002 B.E. Van Wyk, A review of ethnobotanical research in southern Africa, S. Afr. J. Bot. 68 (2002), pp. 1-13.

Velíšek et al., 2006 J. Velíšek, R. Kubec and K. Cejpek, Biosynthesis of food constituents: amino acids. 4. Non-protein amino acids-a review, Czech J. Food Sci. 24 (2006), pp. 93109.

Williams, 1999 R.B. Williams, A compartmentalized model for the estimation of the cost of coccidiosis to the world's chicken production industry, Int. J. Parasitol. 29 (1999), pp. 12091229.

Yilmaz and Toledo, 2004 Y. Yilmaz and R.T. Toledo, Health aspects of functional grape seed constituents, Trends Food Sci. Technol. 15 (2004), pp. 422-433.

Youn and Noh, 2001 H.J. Youn and J.W. Noh, Screening of the anticoccidial effects of herb extracts against Eimeria tenella, Vet. Parasitol. 96 (2001), pp. 257-263.

Zheng and Wang, $2001 \mathrm{~W}$. Zheng and S.Y. Wang, Antioxidant activity and phenolic compounds in selected herbs, J. Agric. Food Chem. 49 (2001), pp. 5165-5170.

Zishiri, 2004 Zishiri, V.K., 2004. Potentising and application of a Combretum woodii leaf extract with high antibacterial and antioxidant activity. MSc Thesis, University of Pretoria. 